\title{
A NONLINEAR ELECTROMECHANICAL PENDULUM ARM WITH A NONLINEAR ENERGY SINK CONTROL (NES) APPROACH
}

\author{
G. Füsun Alişveriş̧ı, HÜseyin BayiroĞLu \\ YıldızTechnical University, Istanbul, Turkey; e-mail: afusun@yildiz.edu.tr; hbayir@yildiz.edu.tr \\ Jorge Luis Palacios Felix \\ UFFS - Federal University of Fronteira Sul, Cerro Largo, RS, Brazil; e-mail: jorge.felix@uffs.edu.br \\ José M. BALthazAR \\ ITA - Aeronautics Technological Institute, São José dos Campos, SP, Brazil and UNESP-Bauru, SP, Brazil \\ e-mail: jmbaltha@gmail.com \\ Reyolando Manoel Lopes Rebello da Fonseca Brasil \\ Federal University of ABC, Santo André, SP, Brazil; e-mail: reyolando.brasil@ufabc.edu.br
}

\begin{abstract}
This paper considers the nonlinear dynamics of an electromechanical device with a pendulum arm and a Nonlinear Energy Sink (NES) put on the point of the pendulum suspension. It is shown that the (NES) is capable of absorbing energy from the system. The numerical results are shown in a bifurcation diagram, phase plane, Poincaré map and Lyapunov exponents.
\end{abstract}

Keywords: nonlinear electromechanical system, bifurcations, nonlinear energy sink

\section{Introduction}

Mechanical oscillatory systems (e.g., a pendulum system which is a basic subsystem of any robot) are of special interest for research and applications as examples of simple systems that may exhibit complex nonlinear behavior. That is why mechanical pendulum-like models of robot arms and mechanical manipulators have recently drawn attention of researchers (Mogo and Woafo, 2007). An interesting example of a driven pendulum device coupled with an electric circuit through a magnetic field. This enters the class of nonlinear electromechanical devices with a pendulum arm. It has been found that the device displays different nonlinear behavior, including chaos (Mogo and Woafo, 2007). SDRE control and sensibility analysis of a chaotic double pendulum arm excited by a RLC circuit based nonlinear shaker was presented by Tusset et al. (2014, 2015). A non-ideal electromechanical damping vibration absorber, the Sommerfeld effect and energy transfer were studied by Felix and Balthazar (2009). The energy pumping, synchronization and beat phenomenon in a non-ideal structure coupled with an essentially nonlinear oscillator were discussed by Felix et al. (2009).

This paper deals with a thin rod mounted to a plate to which electrical windings are applied. Connected to an electric circuit (Tusset et al., 2014, 2015), its oscillations are due to the electromagnetic force resulting from two identical and repulsive permanent magnets (Mogo and Woafo, 2007) as well as a Nonlinear Energy Sink. NES has recently drawn attention of many researchers. The NES method represents a new and unique application of strong nonlinearity. The nonlinear energy sink is a local, simple, lightweight subsystem capable of completely altering the global behavior of the primary system to which it is attached (Vakakis et al., 2008). Elimination of chaotic behavior in a non-ideal portal frame structural system using both passive and active controls were described by Tusset et al. (2013). Steady-state dynamics of a linear 
structure weakly coupled to an essentially nonlinear oscillator was studied by Malatkar and Nayfeh (2007).

Steady state passive nonlinear energy pumping in coupled oscillators was studied by Jiang et al. (2003). Introduction of passive nonlinear energy sinks to linear systems was discussed by Vakakis (2001). Energy pumping in nonlinear mechanical oscillators for resonance capture was examined by Vakakis and Gendelman (2001). The energy transfer between linear and non-linear oscillators was investigated by Dantas and Balthazar (2008).
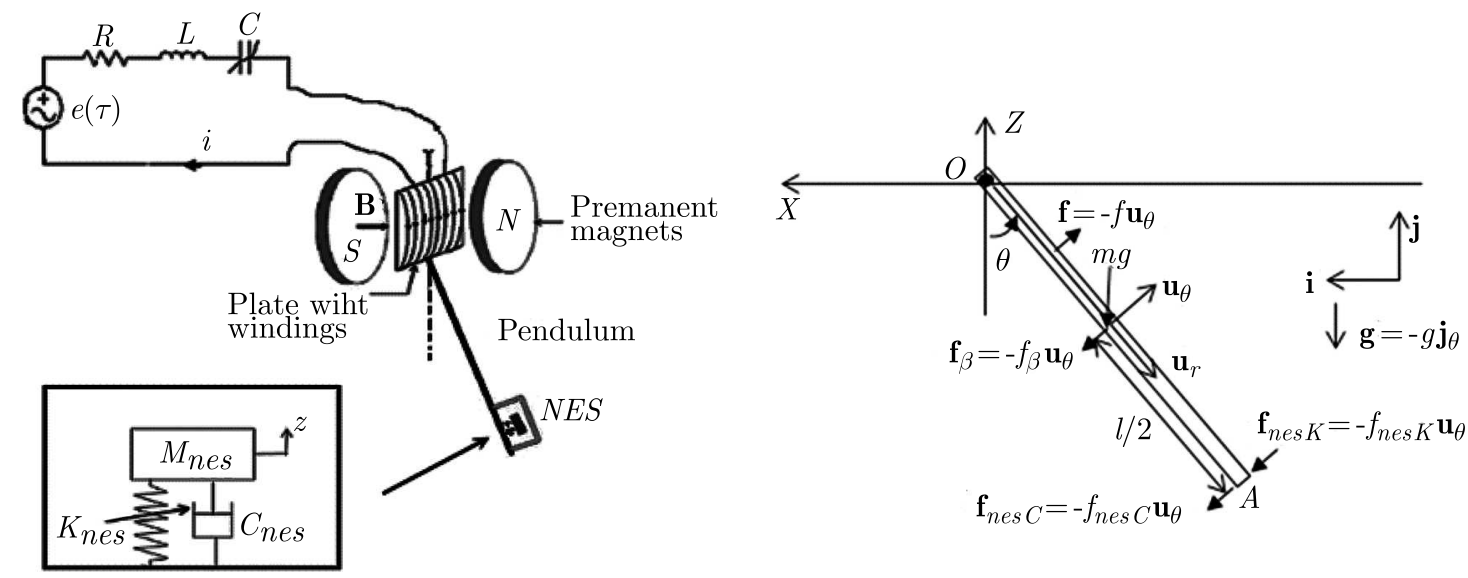

Fig. 1. Idealization of a NES applied to a pendulum arm, $\mathbf{u}_{r}$ and $\mathbf{u}_{\theta}$ are the polar unit vectors

In this work, a NES device is applied to the free end of the pendulum (Fig. 1). This set-up is a system with three degrees of freedom: (i) charge $q$ of the nonlinear condenser, (ii) angular displacement $\theta$ of the pendulum, (iii) displacement $\zeta$ of the $M_{\text {nes }}$.

\section{Equations of motion}

\subsection{Equation of the electric drive}

The electric oscillator used to drive the pendulum is an RLC series circuit with a sinusoidal excitation $e(t)=v_{0} \cos \Omega t$ ( $v_{0}$ and $\Omega$ being, respectively, the amplitude and frequency, and $t$ time). Denoting the forced mesh current $i$ in the RLC circuit, as shown in Fig. 1, applying Kirchhoff's rules, and taking into account the contribution of Lenz's electromotive voltage for $N$ turns by integrating over $\theta$ from zero to $2 \pi N$ ( $\left.e=-0.5 N B \sigma^{2} l^{2} d \theta / d t\right)$ one obtains

$$
L \frac{d i}{d t}+R i+V_{c}(q)-e=e(t)
$$

where $L d i / d t, R i, V_{C}(q)$ are the voltages across the selenoid of the inductance $L$, the resistor $R$, and the nonlinear capacitor $C$, respectively. In this electromechanical model, the electrical nonlinear term is introduced by considering that the voltage of the capacitor is a nonlinear function of the instantaneous electrical charge $q$ of the following form

$$
V_{c}(q)=\frac{1}{C_{0}} q+a_{3} q^{3}
$$

where $C_{0}$ is the linear value of $C$ and $a_{3}$ is the nonlinear coefficient depending on the type of the capacitor used. Inserting Eq. (2.2) in Eq. (2.1), the electric part of the model is described by the following nonlinear differential equation

$$
\frac{d^{2} q}{d t^{2}}+\frac{R}{L} \frac{d q}{d t}+\omega_{e}^{2} q+\frac{a_{3}}{L} q^{3}+\frac{N B \sigma^{2} l^{2}}{2 L} \frac{d \theta}{d t}=\frac{v_{0}}{L} \cos (\Omega t)
$$

where $\omega_{e}^{2}=1 /\left(L C_{0}\right)$ is the resonance frequency of the electric oscillator. 


\subsection{Equation of the pendulum arm and the Nonlinear Energy Sink}

In Fig. 1, the forces acting on the pendulum are represented. The mechanism consists of a uniform thin rod $O A$ of mass $m$ and length $l$, having a plate to which $N$ conducting electric windings of length $\sigma l$ each are applied, with $\sigma=1 / 2$. The pendulum is hinged at $O$ about a horizontal axis, with only the portion $\sigma l$ of the rod in the magnetic field. The total mass of the conducting wire and the plate bathing in the magnetic field have been neglected compared to the pendulum mass. The moment of inertia of the output is then reduced to

$$
I_{O}=\frac{1}{3} m l^{2}+M_{n e s} l^{2}
$$

where $m, M_{n e s}$ are mass of the pendulum and NES, respectively. When the current $i$ flows through the conducting wire in the magnetic field, there appear, according to the directions of the current (upward or downward), two identical Laplace forces (direction and intensity) whose resultant $f$ sets the pendulum into motion in a viscous medium with the frictional coefficient $\beta$. According to the equation for kinetic moment, the moment of inertia $I_{O}$ times the angular acceleration equals the sum of torques due to forces applied to the pendulum. The Laplace force $f=N B \sigma l d q / d t$ is applied to the center of the plate gravity and friction forces. NES spring and friction forces are applied to the free end of the pendulum.

Thus, the pendulum motion is described by

$$
I_{O} \frac{d^{2} \theta}{d t^{2}}=\frac{N B \sigma^{2} l^{2}}{2} \frac{d q}{d t}-\frac{m g l}{2} \sin \theta-\frac{\beta l^{2}}{4} \frac{d \theta}{d t}-C_{n e s} l\left(l \frac{d \theta}{d t}-\frac{d \zeta}{d t}\right)-K_{n e s} l(l \theta-\zeta)^{n}
$$

where $n=1$ and $n=3$. It can be divided by the moment of inertia to obtain this equation in the standard form

$$
\begin{aligned}
\frac{d^{2} \theta}{d t^{2}} & =\frac{N B \sigma^{2} l^{2}}{2\left(\frac{m}{3}+M_{n e s}\right) l^{2}} \frac{d q}{d t}-\frac{m g l}{2\left(\frac{m}{3}+M_{n e s}\right) l^{2}} \sin \theta-\frac{\beta l^{2}}{4\left(\frac{m}{3}+M_{n e s}\right) l^{2}} \frac{d \theta}{d t} \\
& -\frac{C_{n e s} l}{\left(\frac{m}{3}+M_{n e s}\right) l^{2}}\left(l \frac{d \theta}{d t}-\frac{d \zeta}{d t}\right)-\frac{K_{n e s} l}{\left(\frac{m}{3}+M_{n e s}\right) l^{2}}(l \theta-\zeta)^{n} \\
\frac{d^{2} \theta}{d t^{2}} & +\omega_{m}^{2} \sin \theta+\frac{\beta}{4\left(\frac{m}{3}+M_{n e s}\right)} \frac{d \theta}{d t}-\frac{N B \sigma^{2}}{2\left(\frac{m}{3}+M_{n e s}\right)} \frac{d q}{d t}+\frac{C_{n e s}}{\left(\frac{m}{3}+M_{n e s}\right) l}\left(l \frac{d \theta}{d t}-\frac{d \zeta}{d t}\right) \\
& +\frac{K_{n e s}}{\left(\frac{m}{3}+M_{n e s}\right) l}(l \theta-\zeta)^{n}=0
\end{aligned}
$$

where $\omega_{m}^{2}=m g /\left[2\left(\frac{m}{3}+M_{n e s}\right) l\right]$ is the resonance frequency of the pendulum, $C_{n e s}$ is the damping coefficients of the NES, $K_{n e s}$ is the spring coefficient of the NES.

According to Newton's second law, the NES motion is described by

$$
M_{n e s} \frac{d^{2} \zeta}{d t^{2}}=-C_{n e s}\left(\frac{d \zeta}{d t}-l \frac{d \theta}{d t}\right)-K_{n e s}(\zeta-l \theta)^{n}
$$

It can be divided by mass of the NES to obtain this equation in the standard form

$$
\frac{d^{2} \zeta}{d t^{2}}+\frac{C_{n e s}}{M_{n e s}}\left(\frac{d \zeta}{d t}-l \frac{d \theta}{d t}\right)+\frac{K_{n e s}}{M_{n e s}}(\zeta-l \theta)^{n}=0
$$




\subsection{Nondimensional equations and values of the parameter}

Using the transformation

$$
\begin{array}{llll}
x=\frac{q}{Q_{0}} & y=\frac{\theta}{\theta_{0}} & z=\frac{\zeta}{l \theta_{0}} & \tau=\omega_{e} t \\
\frac{d q}{d t}=Q_{0} \omega_{e} \frac{d x}{d \tau} & \frac{d \theta}{d t}=\theta_{0} \omega_{e} \frac{d y}{d \tau} & \frac{d \zeta}{d t}=l \theta_{0} \omega_{e} \frac{d z}{d \tau} & \\
\frac{d^{2} q}{d t^{2}}=Q_{0} \omega_{e}^{2} \frac{d^{2} x}{d \tau^{2}} & \frac{d^{2} \theta}{d t^{2}}=\theta_{0} \omega_{e}^{2} \frac{d^{2} y}{d \tau^{2}} & \frac{d^{2} \zeta}{d t^{2}}=l \theta_{0} \omega_{e}^{2} \frac{d^{2} z}{d \tau^{2}} &
\end{array}
$$

where $x, y, \tau$ are dimensionless variables, $Q_{0}$ is the reference charge of the condenser, and $\theta_{0}$ is the reference pendulum angular displacement. The equations of motion of the complete system can be written as follows

$$
\begin{aligned}
& \frac{d^{2} x}{d \tau^{2}}+\frac{R}{L \omega_{e}} \frac{d x}{d \tau}+x+\frac{a_{3} Q_{0}^{2}}{L \omega_{e}^{2}} x^{3}+\frac{N B \sigma^{2} l^{2} \theta_{0}}{2 L Q_{0} \omega_{e}} \frac{d y}{d \tau}=\frac{v_{0}}{Q_{0} \omega_{e}^{2} L} \cos \left(\frac{\Omega}{\omega_{e}} \tau\right) \\
& \frac{d^{2} y}{d \tau^{2}}=\frac{N B \sigma^{2}}{2\left(\frac{m}{3}+M_{n e s}\right) \omega_{e} \theta_{0}} Q_{0} \frac{d x}{d \tau}-\frac{m g}{2\left(\frac{m}{3}+M_{n e s}\right) \theta_{0} \omega_{e}^{2} l} \sin \left(\theta_{0} y\right)-\frac{\beta}{4\left(\frac{m}{3}+M_{n e s}\right) \omega_{e}} \frac{d y}{d \tau} \\
& \quad-\frac{C_{n e s}}{\left(\frac{m}{3}+M_{n e s}\right) \omega_{e}}\left(\frac{d y}{d \tau}-\frac{d z}{d \tau}\right)-\frac{K_{n e s}\left(l \theta_{0}\right)^{n-1}}{\left(\frac{m}{3}+M_{n e s}\right) \omega_{e}^{2}}(y-z)^{n} \\
& \frac{d^{2} z}{d \tau^{2}}+\frac{C_{n e s}}{M_{n e s} \omega_{e}}\left(\frac{d z}{d \tau}-\frac{d y}{d \tau}\right)+\frac{K_{n e s}\left(l \theta_{0}\right)^{n-1}}{M_{n e s} \omega_{e}^{2}}(z-y)^{n}=0
\end{aligned}
$$

and

$$
\begin{aligned}
& x^{\prime \prime}+\mu_{1} x^{\prime}+x+\alpha x^{3}+\gamma_{1} y^{\prime}=E \cos (\omega \tau) \\
& y^{\prime \prime}+\mu_{2} y^{\prime}+\omega_{2}^{2} \sin \left(\theta_{0}^{\prime} y\right)-\gamma_{2} x^{\prime}+c_{2 n e s}\left(y^{\prime}-z^{\prime}\right)+k_{2 n e s}(y-z)^{n}=0 \\
& z^{\prime \prime}+c_{1 n e s}\left(z^{\prime}-y^{\prime}\right)+k_{1 n e s}(z-y)^{n}=0
\end{aligned}
$$

where the prime denotes a derivative with respect to $\tau$ and

$$
\begin{aligned}
& \mu_{1}=\frac{R}{L \omega_{e}} \\
& \alpha=\frac{a_{3} Q_{0}^{2}}{L \omega_{e}^{2}} \\
& E=\frac{v_{0}}{L Q_{0} \omega_{e}^{2}} \\
& \omega=\frac{\Omega}{\omega_{e}} \\
& \gamma_{1}=\frac{N B \sigma^{2} l^{2} \theta_{0}}{2 L Q_{0} \omega_{e}} \\
& \mu_{2}=\frac{\beta}{4\left(\frac{m}{3}+m_{n e s}\right) \omega_{e}} \\
& \omega_{2}^{2}=\frac{\omega_{m}^{2}}{\omega_{e}^{2} \theta_{0}} \\
& \gamma_{2}=\frac{N B \sigma^{2} Q_{0}}{2\left(\frac{m}{3}+m_{n e s}\right) \omega_{e} \theta_{0}} \\
& c_{1 n e s}=\frac{C_{n e s}}{m_{n e s} \omega_{e}} \\
& c_{2 n e s}=\frac{C_{n e s}}{\left(\frac{m}{3}+M_{n e s}\right) \omega_{e}} \\
& k_{1 n e s}=\frac{K_{n e s}\left(l \theta_{0}\right)^{n-1}}{M_{n e s} \omega_{e}^{2}} \\
& k_{2 n e s}=\frac{K_{n e s}\left(l \theta_{0}\right)^{n-1}}{\left(\frac{m}{3}+M_{n e s}\right) \omega_{e}^{2}}
\end{aligned}
$$

The physical parameters used are the following: $C_{0}=0.11 \mathrm{~F}, a_{3}=158 \mathrm{VC}^{-3}, Q_{0}=0.24 \mathrm{C}$, $R=0.97 \Omega, L=1.15 \mathrm{H}, B=0.02 \mathrm{~T}, N=685, \theta_{0}=\pi \mathrm{rad}, l=0.465 \mathrm{~m}, g=9.81 \mathrm{~m} / \mathrm{s}^{2}$, $m=1 \mathrm{~kg}, M_{n e s}=0.1 \mathrm{~kg}, \beta=0.49 \mathrm{Ns} / \mathrm{m}, \Omega=5.6 \mathrm{rad} / \mathrm{s}, \sigma=0.5, K_{n e s}=1.5 \mathrm{~N} / \mathrm{m}$, $C_{n e s}=0.3 \mathrm{Ns} / \mathrm{m}$.

This gives the following values for non-dimensional constants: $\omega=2, \omega_{2}=1, \mu_{1}=0.30$, $\mu_{2}=0.1, \alpha=1, \gamma_{1}=1.5, \gamma_{2}=0.1, c_{1 n e s}=1, c_{2 n e s}=5, k_{1 n e s}=2, k_{2 n e s}=8$. 


\subsection{Bifurcation structures and basin of chaoticity}

The aim of this subsection is to find how chaos arises in our electromechanical model as the parameters of the system evolve. For this purpose, we numerically solve equations of motion (2.11) and plot the resulting bifurcation diagrams, Lyapunov exponents, phase planes and Poincare maps as $E, \omega, \omega_{2}, \alpha, \mu_{1}, \mu_{2}, \gamma_{1}, \gamma_{2}, c_{1 \text { nes }}, c_{2 n e s}, k_{1 \text { nes }}, k_{2 \text { nes }}$ varies. Figures 2 and 3 show the non-dimensional amplitude diagram for the pendulum arm as the other non-dimensional

(a)

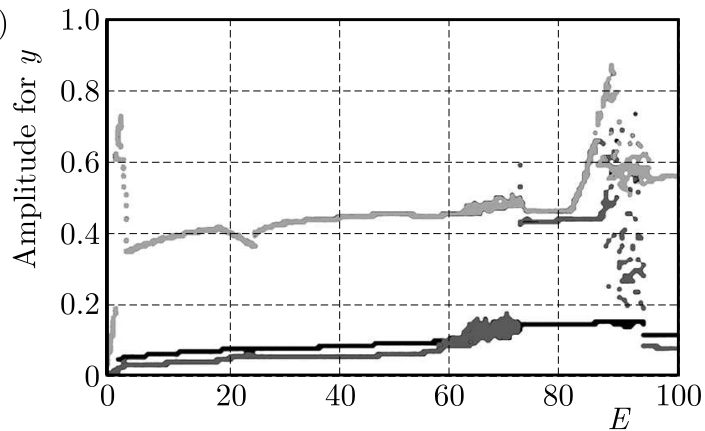

(b)

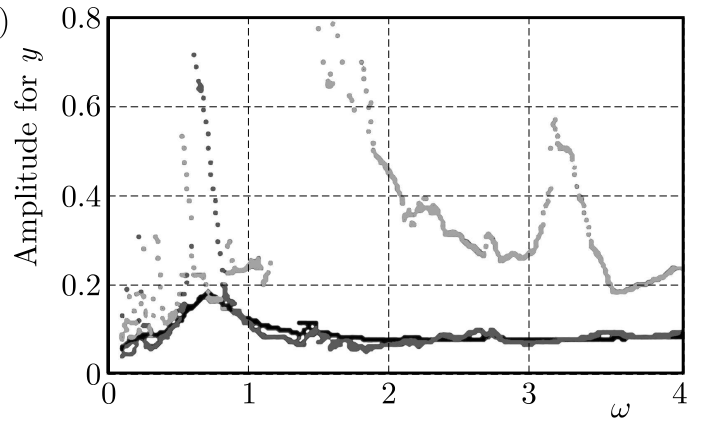

Fig. 2. Amplitude of the pendulum arm versus (a) excitation amplitude $E$, (b) excitation frequency $\omega$; bleu lines $n=1$, black lines $n=3$, grey lines without NES

(a)

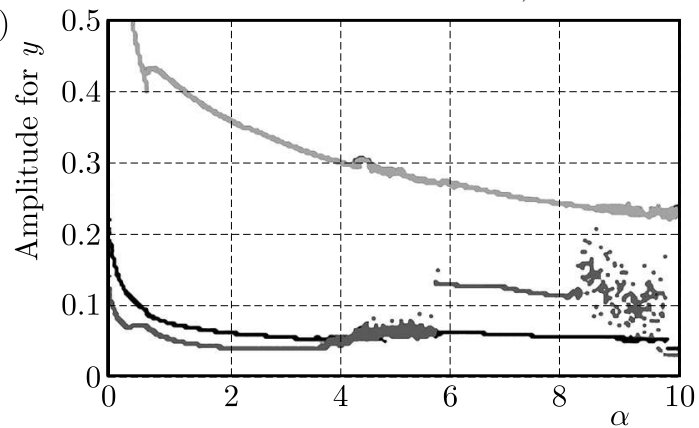

(c)

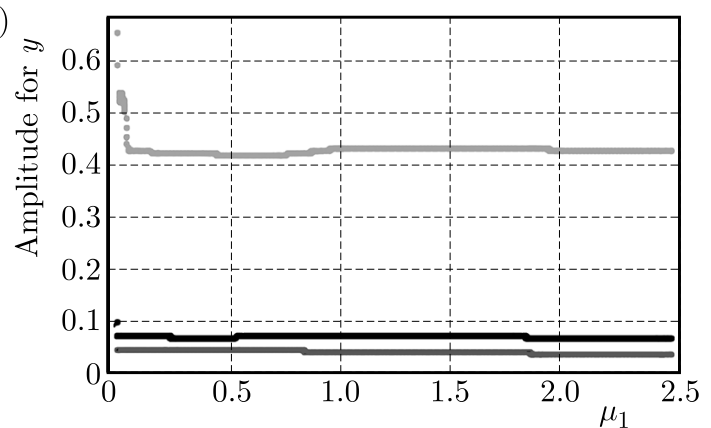

(e)

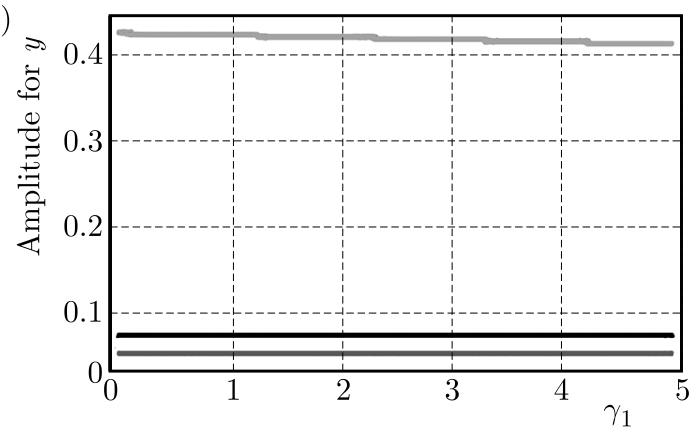

(b)

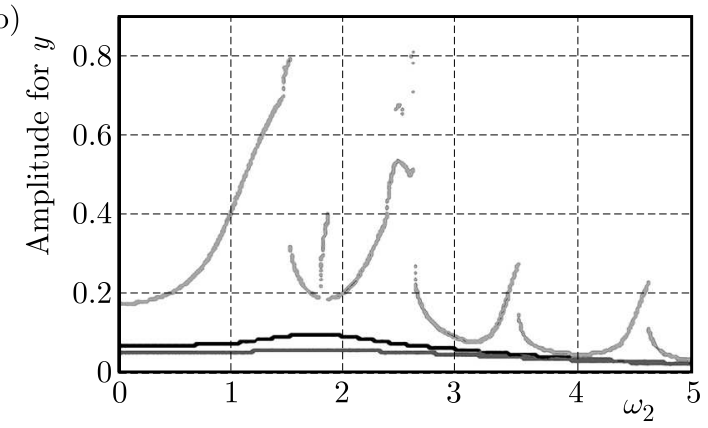

(d)

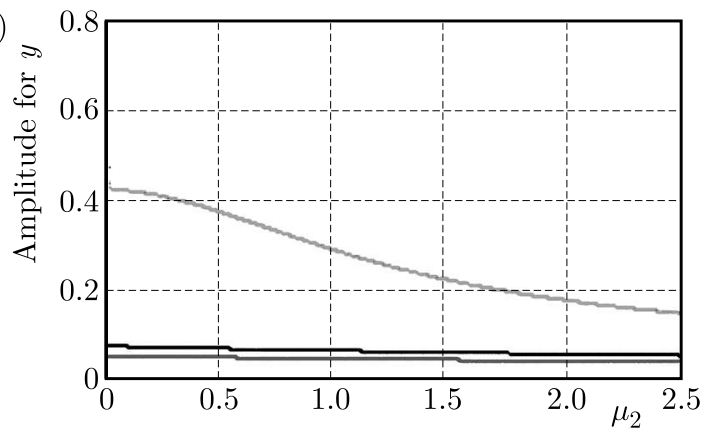

(f)

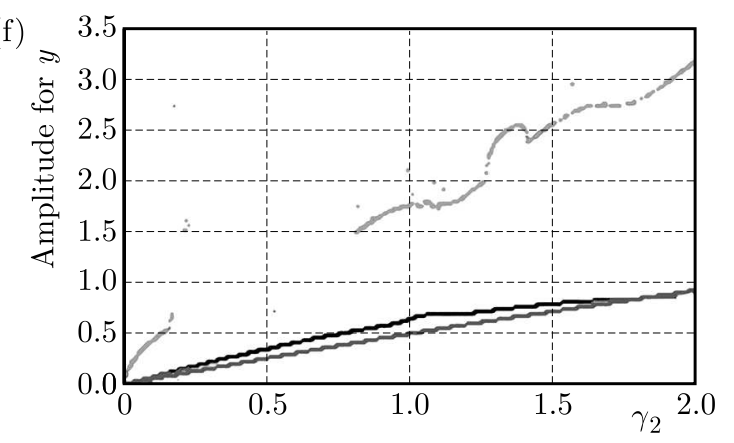

Fig. 3. Amplitude of the pendulum arm versus (a) function of $\alpha$, (b) function of $\omega_{2}$, (c) function of $\mu_{1}$,

(d) function of $\mu_{2}$, (e) function of $\gamma_{1}$, (f) function of $\gamma_{2}$; bleu lines $n=1$, black lines $n=3$, grey lines without NES 
parameters $\left(E, \omega, \omega_{2}, \alpha, \mu_{1}, \mu_{2}, \gamma_{1}, \gamma_{2}\right)$ vary. The dark grey lines are shown for $n=1$, the black lines for $n=3$ and the grey ones for without NES. The investigation of these figures shows that the pendulum arm with NES and $n=3$ diminishes the chaotic effect and amplitude.

Figure 4 shows the non-dimensional amplitude diagram for the pandulum arm as the other non-dimensional parameters $\left(c_{1 \text { nes }}, c_{2 n e s}, k_{1 \text { nes }}, k_{2 n e s}\right)$ vary. The dark grey lines are for $n=1$, the black lines for $n=3$. It is clear that for $n=3$ the chaotic effect and amplitude are diminished.
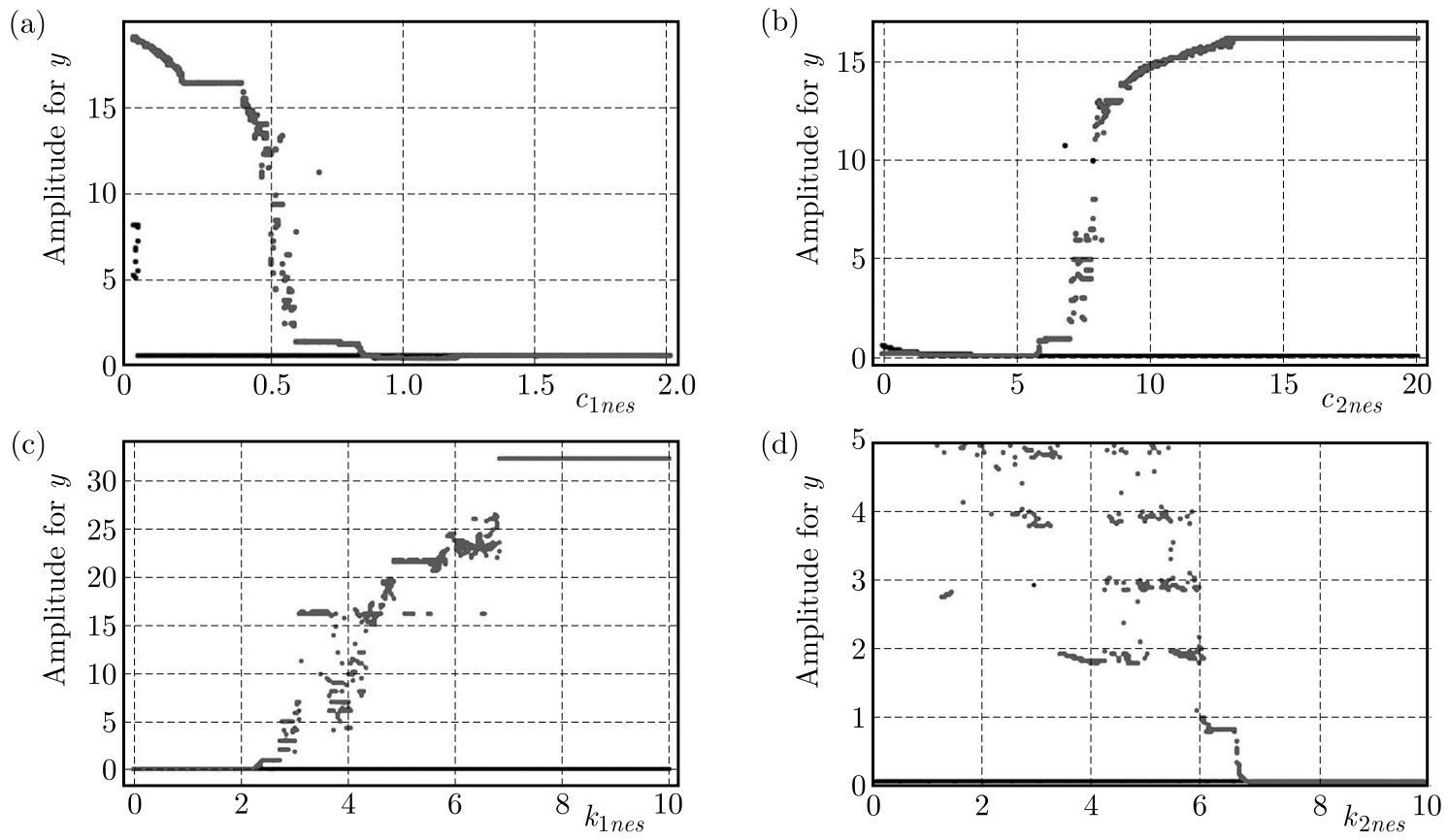

Fig. 4. Amplitude of the pendulum arm versus (a) function of $c_{1 n e s}$, (b) function of $c_{2 n e s}$, (c) function of $k_{1 n e s}$, (d) function of $k_{2 n e s}$; dark grey lines $n=1$, black lines $n=3$

Table 1 shows the stability condition as a function of $E, \omega, \omega_{2}, \alpha, \gamma_{2}, k_{1 \text { nes }}, k_{2 n e s}, c_{1 n e s}, c_{2 n e s}$. It has been constructed with Figs. 5-8 and Figs. 10-14. According to this Table, the pendulum arm with NES and $n=3$ diminishes the chaotic effect and amplitude. Figure 9 shows that only for $\mu 1<0.01$ the system exhibits chaotic effect without NES.
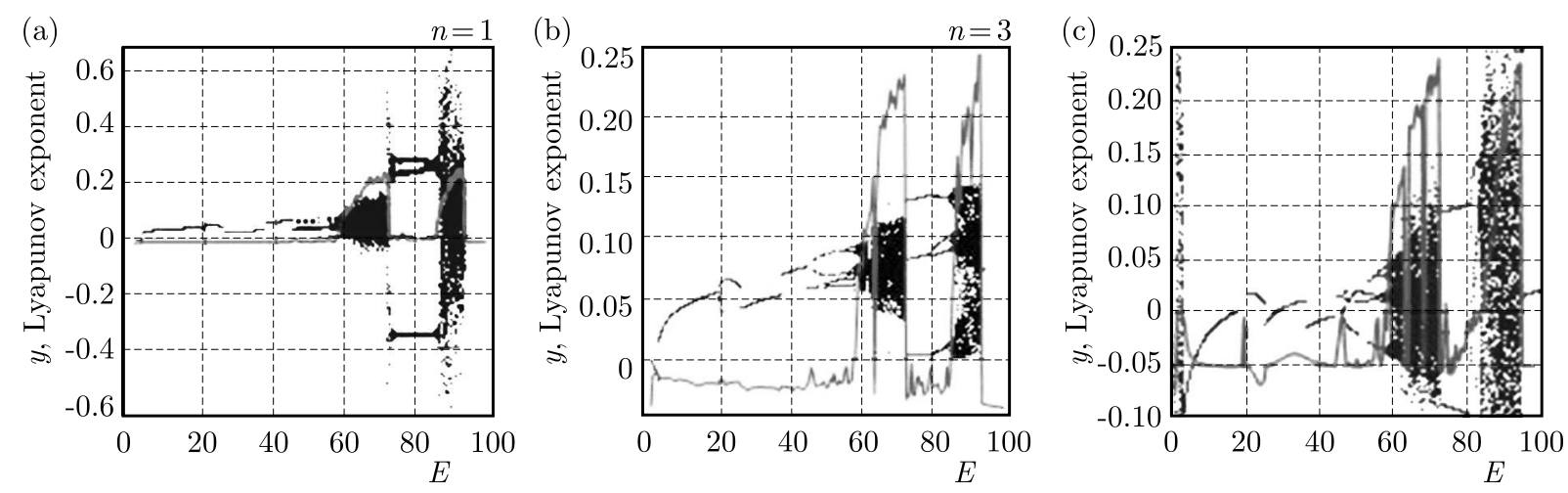

Fig. 5. Bifurcation diagrams and Lyapunov exponents of the pendulum arm as functions of $E$ in the $y$ direction, (a) NES, $n=1$, (b) NES $n=3$, (c) without NES. The other parameters are $E=30, \omega=2$, $\mu_{1}=0.3, \mu_{2}=0.1, \theta_{0}=\pi, \gamma_{1}=1.5, \gamma_{2}=0.1, c_{1 n e s}=1, c_{2 n e s}=5, k_{1 n e s}=2, k_{2 n e s}=8 ;$ dark grey line - bifurcation diagram, grey line - Lyapunov exponent 

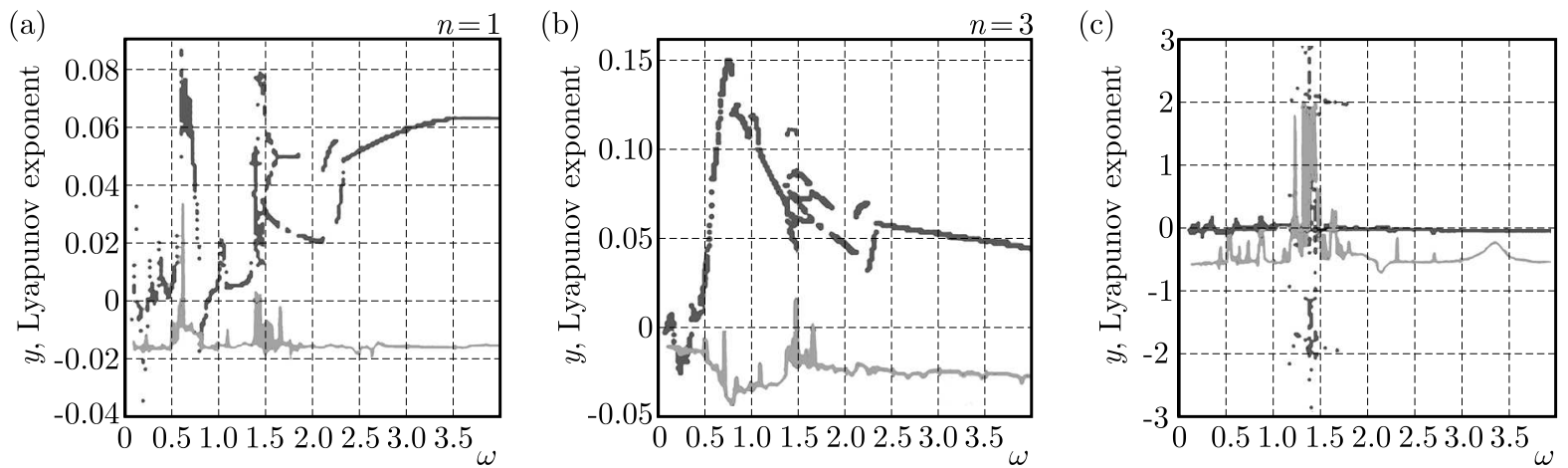

Fig. 6. Bifurcation diagrams and Lyapunov exponents of the pendulum arm as functions of $\omega$ in the $y$ direction, (a) NES, $n=1$, (b) NES $n=3$, (c) without NES. The other parameters are $E=30$, $\omega_{2}=1, \mu_{1}=0.3, \mu_{2}=0.1, \theta_{0}=\pi, \gamma_{1}=1.5, \gamma_{2}=0.1, c_{1 n e s}=1, c_{2 n e s}=5, k_{1 \text { nes }}=2, k_{2 n e s}=8$; dark grey line - bifurcation diagram, grey line - Lyapunov exponent
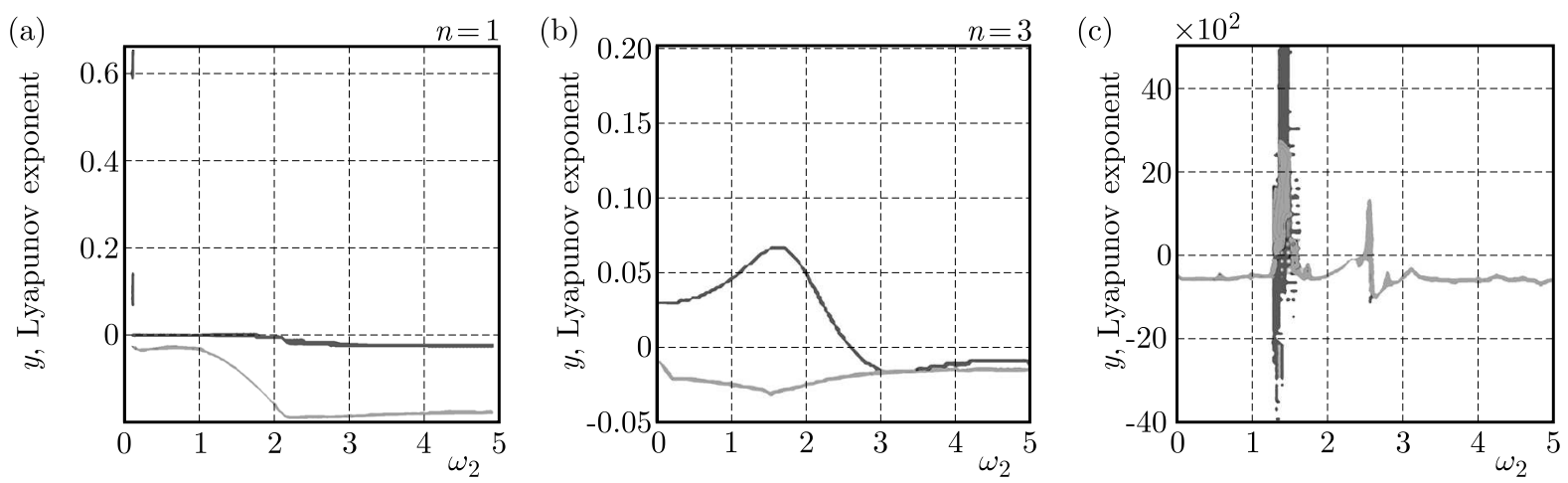

Fig. 7. Bifurcation diagrams and Lyapunov exponents of the pendulum arm as functions of $\omega_{2}$ in the $y$ direction, (a) NES, $n=1$, (b) NES $n=3$, (c) without NES. The other parameters are $E=30, \omega=2$, $\mu_{1}=0.3, \mu_{2}=0.1, \theta_{0}=\pi, \gamma_{1}=1.5, \gamma_{2}=0.1, c_{1 n e s}=1, c_{2 n e s}=5, k_{1 \text { nes }}=2, k_{2 n e s}=8$; dark grey line - bifurcation diagram, grey line - Lyapunov exponent
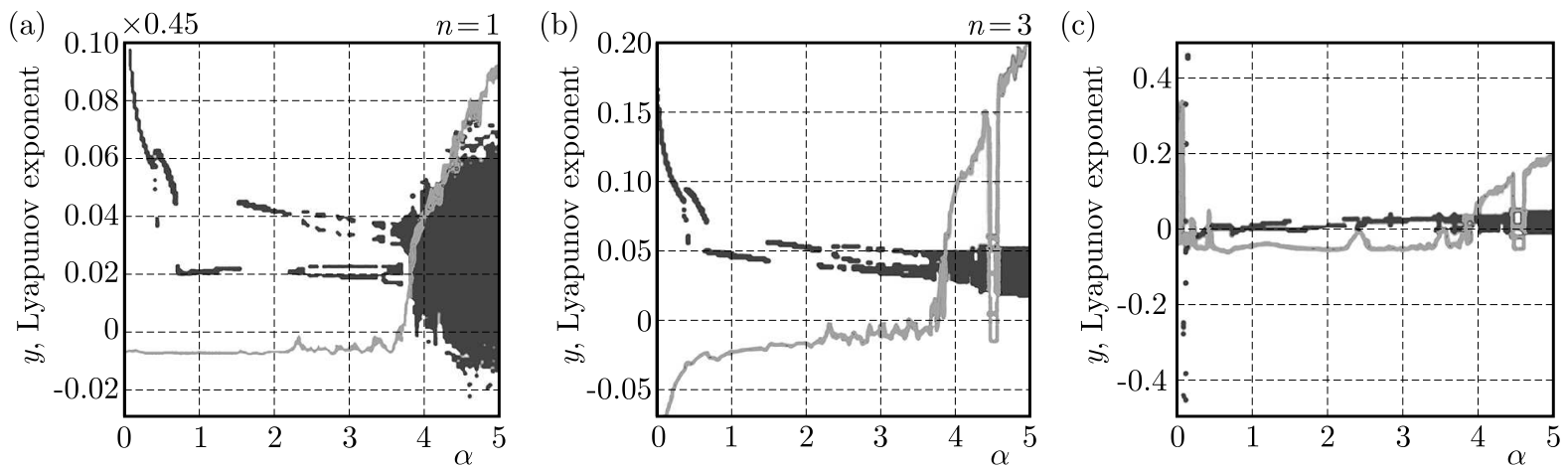

Fig. 8. Bifurcation diagrams and Lyapunov exponents of the pendulum arm as functions of $\alpha$ in the $y$ direction, (a) NES, $n=1$, (b) NES $n=3$, (c) without NES. The other parameters are $E=30, \omega=2$, $\mu_{1}=0.3, \mu_{2}=0.1, \theta_{0}=\pi, \gamma_{1}=1.5, \gamma_{2}=0.1, c_{1 n e s}=1, c_{2 n e s}=5, k_{1 \text { nes }}=2, k_{2 n e s}=8$; dark grey line - bifurcation diagram, grey line - Lyapunov exponent 

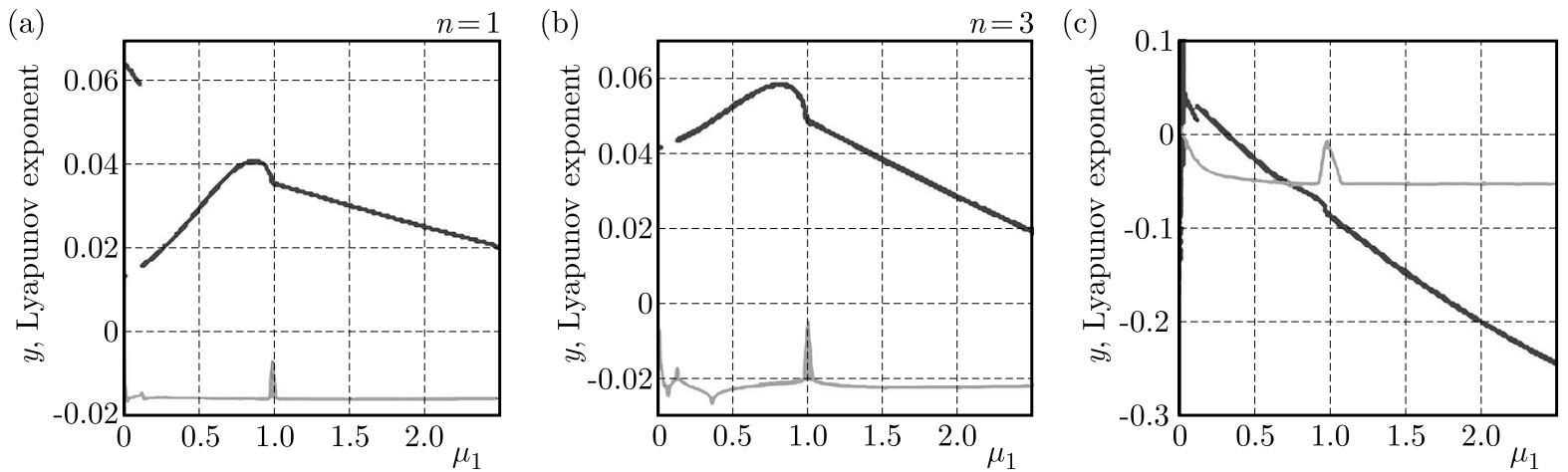

Fig. 9. Bifurcation diagrams and Lyapunov exponents of the pendulum arm as functions of $\mu_{1}$ in the $y$ direction, (a) NES, $n=1$, (b) NES $n=3$, (c) without NES. The other parameters are $E=30, \omega=2$, $\alpha=1, \mu_{2}=0.1, \theta_{0}=\pi, \gamma_{1}=1.5, \gamma_{2}=0.1, c_{1 n e s}=1, c_{2 n e s}=5, k_{1 n e s}=2, k_{2 n e s}=8$;

dark grey line - bifurcation diagram, grey line - Lyapunov exponent
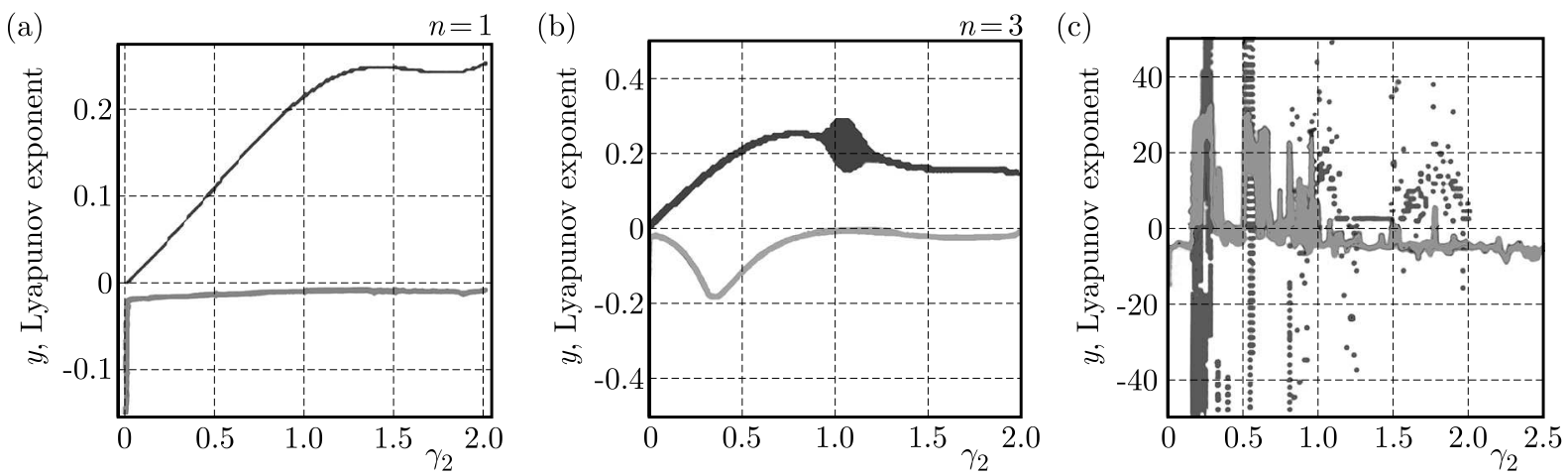

Fig. 10. Bifurcation diagrams and Lyapunov exponents of the pendulum arm as functions of $\gamma_{2}$ in the $y$ direction, (a) NES, $n=1$, (b) NES $n=3$, (c) without NES. The other parameters are $E=30, \omega=2$, $\mu_{1}=0.3, \mu_{2}=0.1, \theta_{0}=\pi, \alpha=1, \gamma_{1}=1.5, c_{1 \text { nes }}=1, c_{2 n e s}=5, k_{1 \text { nes }}=2, k_{2 n e s}=8$; dark grey line - bifurcation diagram, grey line - Lyapunov exponent

(a)

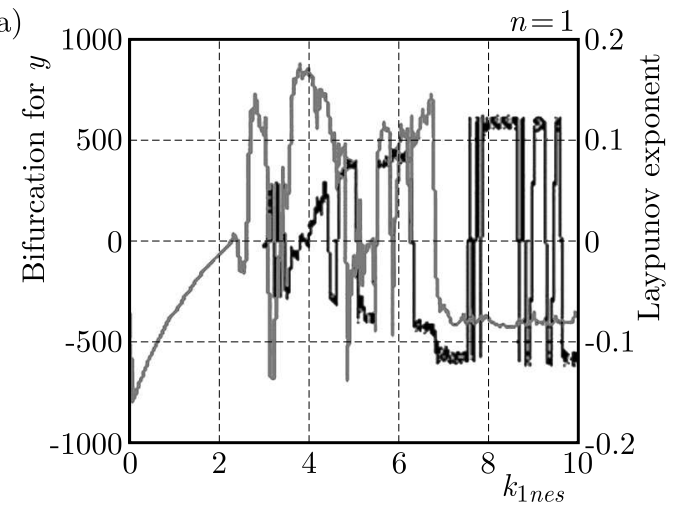

(b)

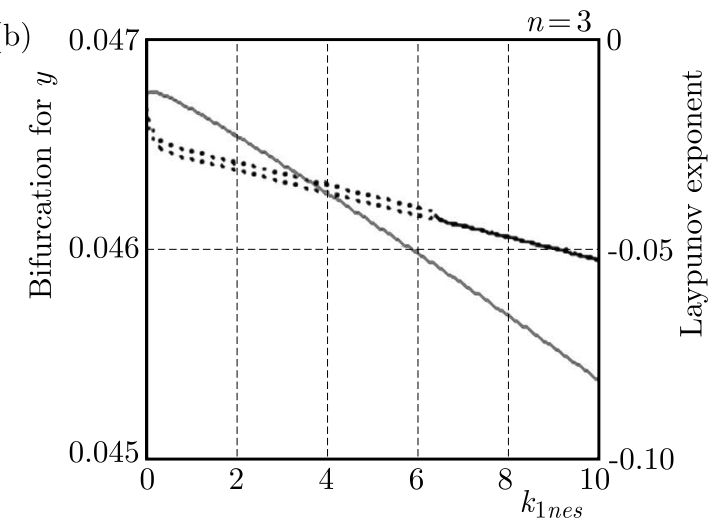

Fig. 11. Bifurcation diagrams and Lyapunov exponents of the pendulum arm as functions of $k_{1 \text { nes }}$ with NES, (a) $n=1$, (b) $n=3$. The other parameters are $E=30, \omega=2, \mu_{1}=0.3, \mu_{2}=0.1, \theta_{0}=\pi, \alpha=1$, $\gamma_{2}=0.1, c_{1 n e s}=1, c_{2 n e s}=5, k_{2 n e s}=8$; dark grey line - bifurcation diagram, grey line - Lyapunov exponent 
(a)

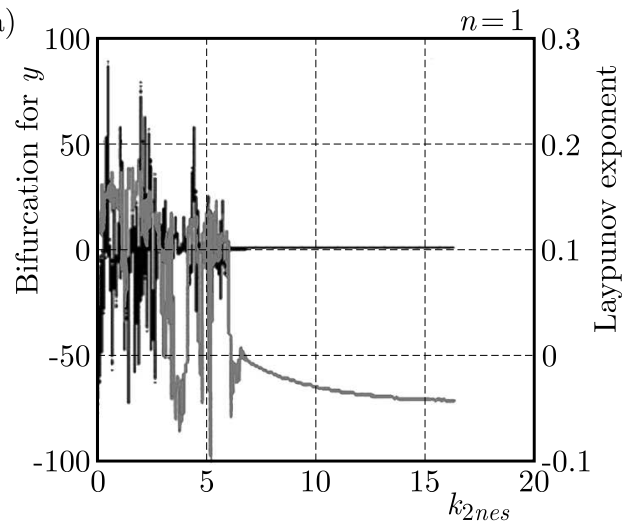

(b)

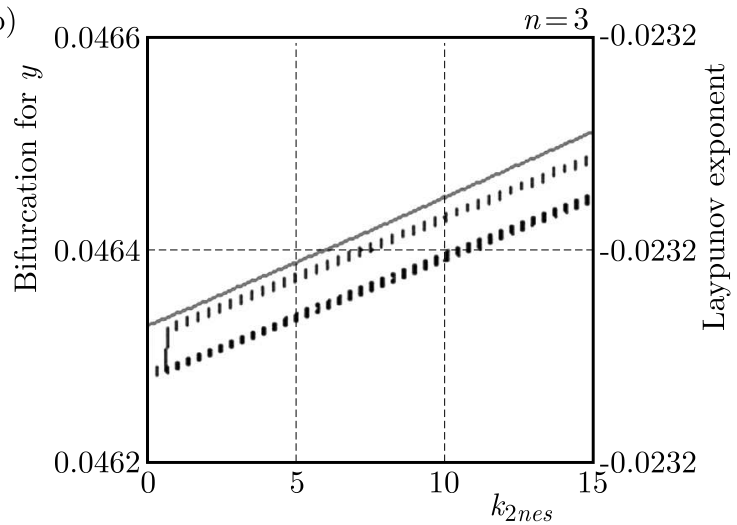

Fig. 12. Bifurcation diagrams and Lyapunov exponents of the pendulum arm as functions of $k_{2 n e s}$ with NES, (a) $n=1$, (b) $n=3$. The other parameters are $E=30, \omega=2, \mu_{1}=0.3, \mu_{2}=0.1, \theta_{0}=\pi, \alpha=1$, $\gamma_{2}=0.1, c_{1 n e s}=1, c_{2 n e s}=5, k_{1 n e s}=2$; dark grey line - bifurcation diagram, grey line - Lyapunov exponent

(a)

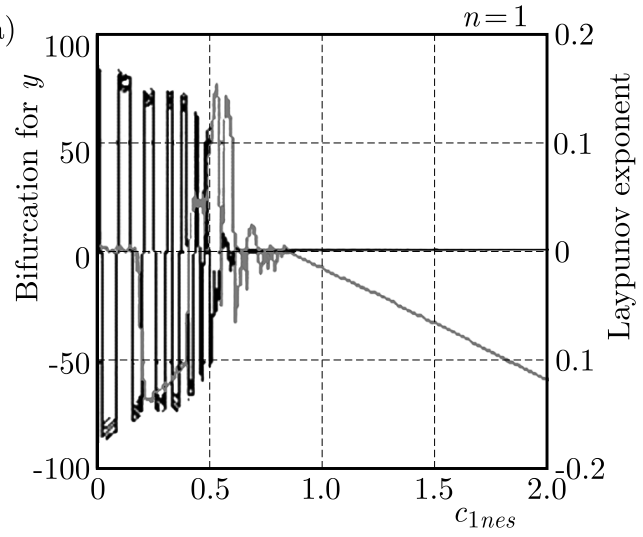

(b)

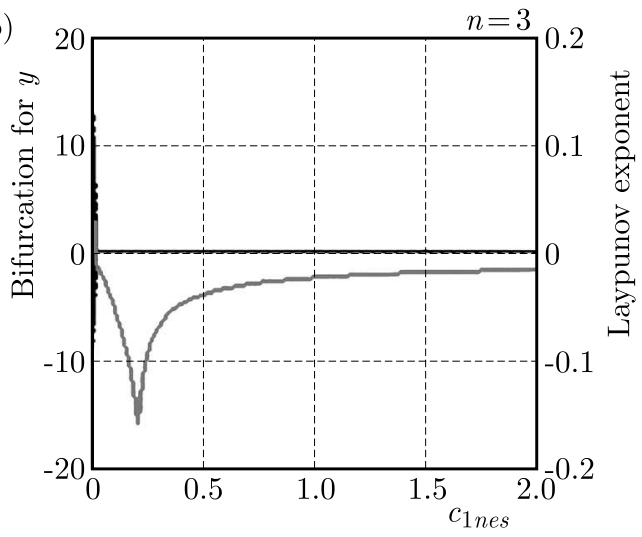

Fig. 13. Bifurcation diagrams and Lyapunov exponents of the pendulum arm as functions of $c_{1 \text { nes }}$ with NES, (a) $n=1$, (b) $n=3$. The other parameters are $E=30, \omega=2, \mu_{1}=0.3, \mu_{2}=0.1, \theta_{0}=\pi, \alpha=1$, $\gamma_{2}=0.1, c_{1 n e s}=1, c_{2 n e s}=5, k_{1 n e s}=2, k_{2 n e s}=8$; dark grey line - bifurcation diagram, grey line - Lyapunov exponent

(a)

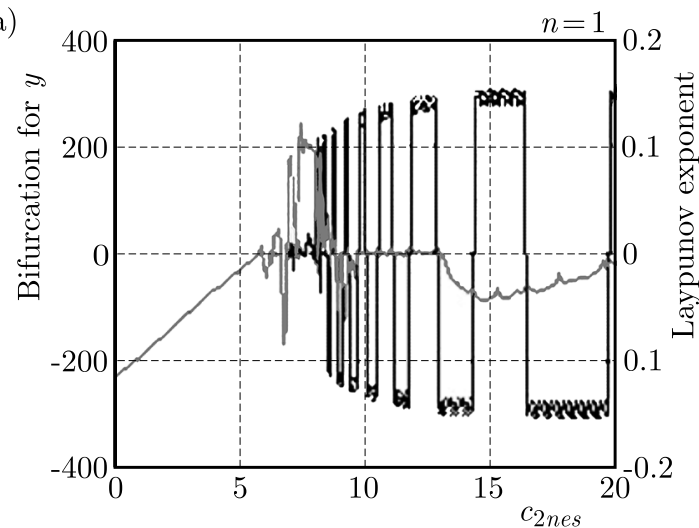

(b)

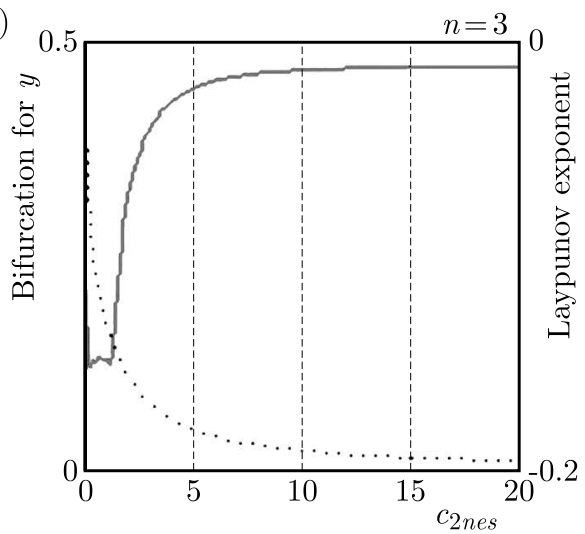

Fig. 14. Bifurcation diagrams and Lyapunov exponents of the pendulum arm as functions of $c_{2 n e s}$ with NES, (a) $n=1$, (b) $n=3$. The other parameters are $E=30, \omega=2, \mu_{1}=0.3, \mu_{2}=0.1, \theta_{0}=\pi, \alpha=1$, $\gamma_{2}=0.1, c_{1 n e s}=1, k_{1 n e s}=2, k_{2 n e s}=8$; dark grey line - bifurcation diagram, grey line - Lyapunov exponent 
Table 1. Stability conditions as a function of $E, \omega, \omega_{2}, \alpha, \gamma_{2}, k_{1 n e s}, k_{2 n e s}, c_{1 n e s}, c_{2 n e s}$

\begin{tabular}{|c|c|c|c|}
\hline & Periodic & Quasi periodic & Chaotic \\
\hline $\begin{array}{l}\text { Fig. 5a, } n=1 \text {, } \\
\text { various } E\end{array}$ & $\begin{array}{l}E<45 \\
E>93\end{array}$ & $\begin{array}{l}45<E<57, \\
73<E<87\end{array}$ & $\begin{array}{l}57<E<73, \\
87<E<93\end{array}$ \\
\hline $\begin{array}{l}\text { Fig. } 5 \mathrm{~b}, n=3 \text {, } \\
\text { various } E\end{array}$ & $\begin{array}{c}E<20 \\
93<E \\
28<E<45\end{array}$ & $\begin{array}{l}20<E<28 \\
45<E<58 \\
72<E<86\end{array}$ & $\begin{array}{l}58<E<72, \\
86<E<93\end{array}$ \\
\hline $\begin{array}{l}\text { Fig. 5c, without } \\
\text { NES, various } E\end{array}$ & $\begin{array}{c}5<E<20 \\
E>95\end{array}$ & $\begin{array}{c}E=32 \\
48<E<60\end{array}$ & $\begin{array}{c}E<5 \\
60<E<73 \\
85<E<95\end{array}$ \\
\hline $\begin{array}{l}\text { Fig. } 6 \text { a, } n=1 \text {, } \\
\text { various } \omega\end{array}$ & $\begin{array}{l}\omega<0.7 \\
\omega>2.3\end{array}$ & $\begin{array}{l}1.5<\omega<1.8, \\
2.1<\omega<2.3\end{array}$ & $\begin{array}{l}0.7<\omega<0.8 \\
1.3<\omega<1.45\end{array}$ \\
\hline $\begin{array}{l}\text { Fig. } 6 \mathrm{~b}, n=3 \text {, } \\
\text { various } \omega\end{array}$ & $\begin{array}{c}\omega<1.45 \\
\omega>2.3\end{array}$ & $1.5<\omega<1.7$ & $\begin{array}{c}1.45<\omega<1.5, \\
\omega=1.7\end{array}$ \\
\hline $\begin{array}{l}\text { Fig. 6c, without } \\
\text { NES, various } \omega\end{array}$ & $\begin{array}{l}\omega<0.4 \\
\omega>2.3\end{array}$ & $\begin{array}{c}0.5<\omega<0.6 \\
0.8<\omega<0.9\end{array}$ & $\begin{array}{l}1.2<\omega<1.5 \\
1.6<\omega<1.7\end{array}$ \\
\hline $\begin{array}{l}\text { Fig. } 7 \mathrm{a}, n=1, \\
\text { various } \omega_{2}\end{array}$ & $0>\omega_{2}<5$ & & \\
\hline $\begin{array}{l}\text { Fig. } 7 \mathrm{~b}, n=3, \\
\text { various } \omega_{2}\end{array}$ & $0>\omega_{2}<5$ & & \\
\hline $\begin{array}{l}\text { Fig. 7c, without } \\
\text { NES, various } \omega_{2}\end{array}$ & $\begin{array}{c}\omega_{2}<1.3 \\
\omega_{2}>2.55 \\
1.6<\omega_{2}<2.5\end{array}$ & & $\begin{array}{l}1.3<\omega_{2}<1.6 \\
2.5<\omega_{2}<2.55\end{array}$ \\
\hline $\begin{array}{l}\text { Fig. } 8 \mathrm{a}, n=1 \text {, } \\
\text { various } \alpha\end{array}$ & $\alpha<2.3$ & $2.3<\alpha<3.7$ & $\alpha>3.7$ \\
\hline $\begin{array}{l}\text { Fig. } 8 \mathrm{~b}, n=3 \text {, } \\
\text { various } \alpha\end{array}$ & $\alpha<2.2$ & $\begin{array}{c}2.3<\alpha<3.7 \\
4.4<\alpha<4.6\end{array}$ & $\begin{array}{c}3.7<\alpha<4.4 \\
\alpha>4.6\end{array}$ \\
\hline $\begin{array}{l}\text { Fig. 8c, without } \\
\text { NES, various } \alpha\end{array}$ & $\begin{array}{c}0.2<\alpha<0.4 \\
0.5<\alpha<1 \\
1.4<\alpha<2.2\end{array}$ & $\begin{array}{l}1.2<\alpha<1.4 \\
2.2<\alpha<3.4 \\
4.4<\alpha<4.6\end{array}$ & $\begin{array}{c}0<\alpha<0.2 \\
0.4<\alpha<0.5 \\
3.4<\alpha<3.6 \\
3.8<\alpha<4.4 \\
4.6<\alpha<5\end{array}$ \\
\hline $\begin{array}{l}\text { Fig. 10a, } n=1 \text {, } \\
\text { various } \gamma_{2}\end{array}$ & $0<\gamma_{2}<2$ & & \\
\hline $\begin{array}{l}\text { Fig. } 10 \mathrm{~b}, n=3, \\
\text { various } \gamma_{2}\end{array}$ & $\begin{array}{c}0<\gamma_{2}<0.8 \\
1.38<\gamma_{2}<2\end{array}$ & & $0.8<\gamma_{2}<1.3$ \\
\hline $\begin{array}{l}\text { Fig. 10c, without } \\
\text { NES, various } \gamma_{2}\end{array}$ & $\begin{array}{c}0<\gamma_{2}<0.15 \\
1.35<\gamma_{2}<1.4\end{array}$ & $1.9<\gamma_{2}<2.5$ & $\begin{array}{c}0.15<\gamma_{2}<1.1 \\
1.8<\gamma_{2}<1.9\end{array}$ \\
\hline $\begin{array}{l}\text { Fig. } 11 \mathrm{a}, n=1, \\
\text { various } k_{1 \text { nes }}\end{array}$ & $0<k_{1 \text { nes }}<2.1$ & $\begin{array}{l}3<k_{1 \text { nes }}<3.2 \\
4.8<k_{1 \text { nes }}<5 \\
6.4<k_{1 \text { nes }}<10\end{array}$ & $\begin{array}{l}2.1<k_{1 \text { nes }}<3 \\
3.1<k_{1 \text { nes }}<5 \\
5<k_{1 \text { nes }}<6.4\end{array}$ \\
\hline $\begin{array}{l}\text { Fig. } 11 \mathrm{~b}, n=3 \text {, } \\
\text { various } k_{1 \text { nes }}\end{array}$ & $6.4<k_{1 \text { nes }}<10$ & $0<k_{1 \text { nes }}<6.4$ & \\
\hline $\begin{array}{l}\text { Fig. } 12 \mathrm{a}, n=1 \text {, } \\
\text { various } k_{2 n e s}\end{array}$ & $7<k_{2 n e s}<15$ & & $0<k_{2 n e s}<7$ \\
\hline $\begin{array}{l}\text { Fig. } 12 \mathrm{~b}, n=3 \text {, } \\
\text { various } k_{2 n e s}\end{array}$ & & $0<k_{2 n e s}<15$ & \\
\hline
\end{tabular}




\begin{tabular}{|l|c|c|c|}
\hline $\begin{array}{l}\text { Fig. 13a, } n=1, \\
\text { various } c_{1 \text { nes }}\end{array}$ & $\begin{array}{c}0.2<c_{1 \text { nes }}<0.4, \\
0.7<c_{1 \text { nes }}<2\end{array}$ & $0<c_{1 \text { nes }}<0.2$ & $0.4<c_{1 \text { nes }}<0.7$ \\
\hline $\begin{array}{l}\text { Fig. } 13 \mathrm{~b}, n=3, \\
\text { various } c_{1 \text { nes }}\end{array}$ & $0<c_{1 \text { nes }}<2$ & & \\
\hline $\begin{array}{l}\text { Fig. } 14 \mathrm{a}, n=1, \\
\text { various } c_{2 \text { nes }}\end{array}$ & $0<c_{2 \text { nes }}<2$ & & $2<c_{2 \text { nes }}<20$ \\
\hline $\begin{array}{l}\text { Fig. } 14 \mathrm{~b}, n=3, \\
\text { various } c_{2 \text { nes }}\end{array}$ & $0<c_{2 \text { nes }}<2$ & & $2<c_{2 \text { nes }}<20$ \\
\hline
\end{tabular}

\section{Conclusion}

We have studied the effect of the NES on a electro-mechanical device with a pendulum.

The system exhibits complex dynamical behavior such as multi-periodic, quasi-periodic and chaotic responses, and these are strongly dependent on non-dimensional control parameters $E, \omega$ and a nonlinearity coefficient $\alpha$ for RLC circuit, and NES parameters $c_{1 \text { nes }}, c_{2 n e s}, k_{1 \text { nes }}, k_{2 n e s}$. Moreover, the system without NES exhibits a chaotic response depending on $\gamma_{2}$. The NES parameters $\left(c_{1 n e s}, c_{2 n e s}, k_{1 n e s}, k_{2 n e s}\right)$ for $n=1$ lead to chaotic responses, but for $n=3$, they induce periodic and quasi-periodic responses. It is shown that the NES is capable of absorbing energy from the system and decreases the amplitude as well as diminishes the chaotic effect.

\section{References}

1. Dantas M.J.H., Balthazar J.M., 2008, On energy transfer between linear and non-linear oscillator, Journal of Sound and Vibration, 315, 4/5, 1047-1070

2. Felix J.L.P., Balthazar J.M., 2009, Comments on a nonlinear and a non-ideal electromechanical damping vibration absorber, sommerfeld effect and energy transfer, Nonlinear Dynamics, $\mathbf{5 5}$, $1,1-11$

3. Felix J.L.P., Balthazar J.M., Dantas M.J.H., 2009, On energy pumping, synchronization and beat phenomenon in a non-ideal structure coupled to an essentially nonlinear oscillator, Nonlinear Dynamics, 56, 1, 1-11

4. Jiang X., McFarland D.M., Bergman L.A., Vakakis A.F., 2003, Steady state passive nonlinear energy pumping in coupled oscillators: theoretical and experimental results, Nonlinear Dynamics, 33, 87-102

5. Malatkar P., NAyfen A.H., 2007, Steady-state dynamics of a linear structure weakly coupled to an essentially nonlinear oscillator, Nonlinear Dynamics, 47, 167-179

6. Mogo J.B., WoAfo P., 2007, Dynamics of a nonlinear electromechanical device with a pendulum arm, Journal of Computational and Nonlinear Dynamics, 2, 4, 374-378, DOI: 10.1115/1.2756080

7. Tusset A.M., Balthazar J.M., Felix J.L.P., 2013, On elimination of chaotic behavior in a non-ideal portal frame structural system, using both passive and active controls, Journal of Vibration and Control, 19, 6, 803-813

8. Tusset A.M., Bueno A.M., Sado D., Balthazar J.M., Colón D., 2014, SDRE control and sensibility analysis of a chaotic double pendulum arm excited by a RLC circuit based nonlinear shaker, Conference on Dynamical Systems Theory and Applications, Łódź, Poland, 195-204

9. Tusset A.M., Picirillo V., Bueno A.M., Sado D., Balthazar J.M., Felix J.L.P., 2015, Chaos control and sensitivity analysis of a double pendulum arm excited by an RLC circuit based nonlinear shaker, Journal of Vibration and Control, 1077546314564782 
10. VAKAKIS A.F., 2001, Inducing passive nonlinear energy sinks in linear vibrating systems, Journal of Vibration and Acoustics, 123, 3, 324-332

11. Vakakis F., Gendelman O., 2001, Energy pumping in nonlinear mechanical oscillators. II: Resonance capture, Journal of Applied Mechanics, 68, 1, 42-48

12. Vakakis A.F., Gendelman O.V., Bergman L.A., McFarland D.M., Kerschen G., Lee Y.S., 2008, Nonlinear Targeted Energy Transfer in Mechanical and Structural Systems (Solid Mechanics and Its Applications), Springer

Manuscript received January 13, 2015; accepted for print January 8, 2016 